\title{
New Imaging Modalities in Bone
}

\author{
James F. Griffith • Harry K. Genant
}

Published online: 3 March 2011

(C) The Author(s) 2011. This article is published with open access at Springerlink.com

\begin{abstract}
The digital era has witnessed an exponential growth in bone imaging as new modalities and analytic techniques improve the potential for noninvasive study of bone anatomy, physiology, and pathophysiology. Bone imaging very much lends itself to input across medical and engineering disciplines. It is in part a reflection of this multidisciplinary input that developments in the field of bone imaging over the past 30 years have in some respects outshone those in many other fields of medicine. These developments have resulted in much deeper knowledge of bone macrostructure and microstructure in osteoporosis and a much better understanding of the subtle changes that occur with age, concurrent disease, and treatment. This new knowledge is already being translated into improved day-to day clinical care with better recognition, treatment, and monitoring of the osteoporotic process. As "the more you know, the more you know you don't know" certainly holds true with osteoporosis and bone disease, there is little doubt that further advances in bone imaging and analytical techniques will continue to hold center stage in osteoporosis and related research.
\end{abstract}

J. F. Griffith

Department of Diagnostic Radiology and Organ Imaging,

Chinese University of Hong Kong,

Hong Kong, Hong Kong

H. K. Genant $(\bowtie)$

Departments of Radiology and Medicine,

University of California, San Francisco,

San Francisco, CA 94143, USA

e-mail: harry.genant@ucsf.edu
Keywords Osteoporosis · Bone quality · Radiography . Computed tomography $\cdot$ Magnetic resonance imaging . Image analysis

\section{Introduction}

Within a few months of Rector Wilhelm Conrad Roentgen announcing to the Physico-Medical Society of Würzburg, Germany, on December 28, 1895, that a new type of rays ("x-rays") could penetrate skin and visualize the skeleton, physicians around the world were using this modality to diagnose broken bones and locate bullets within human flesh. Since this enlightened inception, imaging in its many forms has been central to the investigation of bone disease. Particularly during the past three decades, advances in technology have enabled noninvasive bone imaging to evolve dramatically from examination of bone macrostructure and pathology to include the study of bone microstructure, physiology, and pathophysiology [1•].

The aim of osteoporosis screening and treatment is to prevent bone fracture. A fracture occurs when the external force to a bone exceeds its strength. The ability of any bone to resist fracture depends on the amount and distribution of bone as well as its intrinsic properties [2]. The ultimate aims of bone imaging in osteoporosis are to minimize fracture occurrence by identifying the osteoporotic process at an early stage, before significant bone loss occurs; differentiate distinctive patterns of bone loss; accurately predict individual fracture risk; and precisely monitor treatment response. This review introduces some of the new imaging modalities and analytical techniques for bone that have emerged, been enhanced, or been adapted to a new clinical application during the past 3 to 4 years. To put 
these new advances in perspective, we first briefly discuss the background to each imaging modality and the existing clinical application.

\section{Bone Mineral Density Measurement}

\section{Dual X-ray Absorptiometry}

Bone mineral density (BMD) measurement by dual x-ray absorptiometry (DXA) has been available for clinical use since 1987 and is currently the most readily available surrogate marker of bone strength and fracture risk, with fracture risk increasing approximately 1.6-fold for every SD decrement in BMD measurement irrespective of gender [3-5]. DXA is limited in that it assesses only areal BMD (BMDa, in $\mathrm{g} / \mathrm{cm}^{2}$ ) such that, for example, in the spine, vertebral depth is not taken into account. DXA is also limited in that it is a composite measure of both cortical and trabecular bone and prone to overestimation of BMD from degenerative-type hyperostosis.

Recommendations from the International Society for Clinical Densitometry regarding DXA examination for all ages and both genders have been updated [6]. Although DXA is predictive of fracture risk in cohort studies, such epidemiologic evidence cannot be readily applied on an individual patient basis in clinical practice. To address this limitation, individual fracture risk has been standardized using the World Health Organization Fracture Risk Assessment tool (FRAX), which was released in 2009 [7, 8]. FRAX combines BMD from DXA with other risk factors (eg, age, sex, height, previous fracture) to provide a 10-year risk of hip and other major insufficiency fractures $[7,8]$. FRAX is mainly applicable to osteopenic (low bone mass) patients, as the results do not influence the clinical management of patients with normal BMD or osteoporosis [8]. Although it is not an ideal system, FRAX represents an important initiative in allowing clinicians to individualize fracture risk based on DXA examination and other factors [8].

As well as assessing bone density, improvements in fan beam DXA technology have enabled DXA systems to acquire moderately high-resolution images of the thoracic and lumbar spine [9]. These lateral vertebral DXA images are useful for detecting moderate or severe vertebral fractures $(>90 \%$ sensitivity and specificity) but limited in the assessment of mild vertebral fractures [8]. This vertebral fracture assessment provides clear added benefit to the overall assessment by DXA and is recommended in settings in which the result will alter clinical management [6]. In a study of more than 5,000 unselected community-based women 75 years of age or older, lateral vertebral DXA images were sufficient to gauge fracture status in $92 \%$ of vertebrae from $\mathrm{T} 8$ to $\mathrm{L} 3$, and of these women, nearly $15 \%$ had a vertebral fracture. Over a median follow-up period of 4 years, those with prevalent vertebral fracture had a 2.01 relative risk compared with those without vertebral fracture of developing any further osteoporotic fracture, and a 2.29 relative risk for hip fracture. These risks were even greater in patients with more than two prevalent vertebral fractures, increasing to 2.54 and 3.94 , respectively [10].

\section{Quantitative CT}

The value of quantitative $\mathrm{CT}(\mathrm{QCT})$ in allowing volumetric densitometry (measured in $\mathrm{mg} / \mathrm{cm}^{3}$ ) of the trabecular and cortical bony components was recognized in the late-1970s [11-13]. QCT can specifically detect changes in the more metabolically active trabecular bone, making it a more sensitive discriminator of BMD changes than DXA [14]. For example, during a 1-year follow-up of postmenopausal women, a twofold to threefold greater increase in trabecular BMD was shown by QCT in response to parathyroid hormone compared with integral BMD by DXA [15]. The main limitations of QCT regarding BMD measurements are that a clinical CT scanner is required and a larger radiation dose than that with DXA is incurred.

Worldwide, many tens of thousands of thoracic and abdominal CT examinations are performed daily on clinical patients [16]. Several recent studies have shown how it is possible to obtain meaningful QCT BMD data from patients undergoing thoracoabdominal $\mathrm{CT}$ examinations for other clinical reasons without the use of a calibration phantom. Such "phantom-less" BMD data have a high correlation $\left(R^{2}=0.95\right)$ with BMD data obtained from QCT over a wide range of densities [17•]. An internal reference such as subcutaneous fat or paravertebral lean muscle [17•], or a correction factor [18] is used to counteract the lack of an external reference phantom. Phantom-less CT has been studied in patients undergoing noncontrasted CT studies for coronary artery calcium scoring [18], CT colonoscopy, or standard QCT [17•]. Even for contrasted CT examinations, a correction factor adjusting for contrast enhancement can be applied [19]. Phantom-less CT can be applied reasonably to cross-sectional clinical studies, though it should not be used in longitudinal studies, as the precision for phantom-less CT is less than that on standard QCT [17•], especially if contrast has been administered.

These studies demonstrate that it is technically feasible to obtain reasonably accurate QCT BMD spine data in patients undergoing thoracic or abdominal CT examinations for other reasons. Although it may seem appropriate to provide such (unrequested) data as a routine addendum to clinical CT reports, several important practical and clinical questions should be addressed before proceeding down this path [20]. For example, would such 
additional BMD information, which is time consuming and costly to acquire, be of long-term clinical benefit? Would this BMD information be properly managed by the referring clinician (or patient), or would it merely encourage the additional inappropriate use of substantial health resources in terms of clinical management, treatment, and follow-up [20]?

\section{Bone Quality Assessment}

As BMD accounts for only part of the variation seen in bone strength and only some of the observed reduction in fracture risk that occurs with treatment, recent developments have focused more on measuring bone structure and quality of both trabecular and cortical bone rather than bone mass alone. This is done with the knowledge that a measure encompassing bone quality and structure along with bone mass will provide a better prediction of fracture risk than bone mass alone.

\section{Radiography}

Radiography is a low-cost, readily available technique with high spatial resolution capable of providing fine bone detail, especially of distal appendicular skeleton such as the distal forearm and phalanges. Although it is ideally suited for use in large population studies, the limitation of radiography is that as a projectional imaging technique, it cannot consistently visualize individual trabeculae and depends heavily on the depth of tissues under investigation. With increasing sophistication of structural analysis techniques and an improving ability to acquire high-resolution radiographic detail, interest remains in developing radiography to more precisely evaluate trabecular bone detail [21]. A new radiographic device capable of delivering digitized high-resolution radiographs de novo is an advance on previous devices, which required digitization of high-resolution radiographs prior to structural analysis. The number of steps in data analysis is thus reduced, with less potential for introducing error. A new bone texture analysis method known as Laws' masks was applied to these high-resolution digitized radiographs to study trabecular bone architecture in the calcaneus in more than 300 postmenopausal women and was able to distinguish those with fracture from those without fracture independent of BMD and with reasonable reproducibility [21].

\section{Multidetector CT and Volumetric Quantitative CT}

In the 1990s, analytical techniques were applied to highresolution $\mathrm{CT}$ data in a quest to find structural parameters predictive of bone strength. This research gained impetus in the new millennium with the arrival of multidetector CT (MDCT) systems that allowed higher spatial resolution, improved delineation of bone architecture, and faster acquisition of volumetric QCT (vQCT) datasets. Since its inception, the number of detector rows on clinical CT units has increased from 4 to the current clinical standard of 64 rows, although 320-row MDCT systems are also commercially available. Comparing 64-row (in-plane pixel size, $234 \mu \mathrm{m}$; slice increment, $300 \mu \mathrm{m}$ ) and 320-row MDCT (in-plane pixel size, $234 \mu \mathrm{m}$; slice increment, $250 \mu \mathrm{m}$ ) in assessing distal radial trabecular pattern, 320-row MDCT conferred little practical advantage over 64-row MDCT. Both systems yielded different absolute measures, although they still correlated highly $(r=0.92-0.96 ; P<$ 0.0001 ) with a reference standard for bone volume fraction and trabecular spacing. However, as expected, they fared less well with trabecular thickness and number because the spatial resolution of all MDCT systems (250-300 $\mu \mathrm{m})$ remains larger than the trabecular thickness of 50 to $200 \mu \mathrm{m}$ [22]. Nevertheless, structural parameters by MDCT provide a better discriminator of change than DXA. For example, in 65 postmenopausal women, teriparatide increased apparent bone volume fraction by $30.6 \% \pm 4.4 \%$ (mean $\pm \mathrm{SE}$ ), and apparent trabecular number by $19.0 \% \pm$ $3.2 \%$, compared with a $6.4 \% \pm 0.7 \%$ increase in DXAderived BMDa [23].

\section{Medical Image Analysis Framework}

High-precision software known as medical image analysis framework (MIAF) has been developed to facilitate analysis of vQCT datasets [24, 25]. MIAF enables the automatic determination of anatomic coordinates to yield predetermined volume of interests for analysis (Fig. 1). This automated anatomic coordinate system facilitates longitudinal study as well as study of the relative contributions of density, geometry, and trabecular and cortical bone to mechanical failure. Using this advanced image analysis system to study proximal femoral failure load, cortical variables were found to predict as much (59\%) failure load as trabecular variables $(52 \%)$, which was in agreement with previous studies showing that trabecular bone contributes more to subcapital bone strength, while cortical bone contributes more to intertrochanteric bone strength [26]. MIAF can also enable recognition of bone areas most influenced by treatment. For example, ibandronate treatment for 1 year increased volumetric density in the subcortical and extended trabecular areas of the proximal femur, as well as in the extended cortical and superior/ inferior trabecular regions of the vertebral body, all of which are mechanically significant areas $[27 \bullet \bullet]$. 


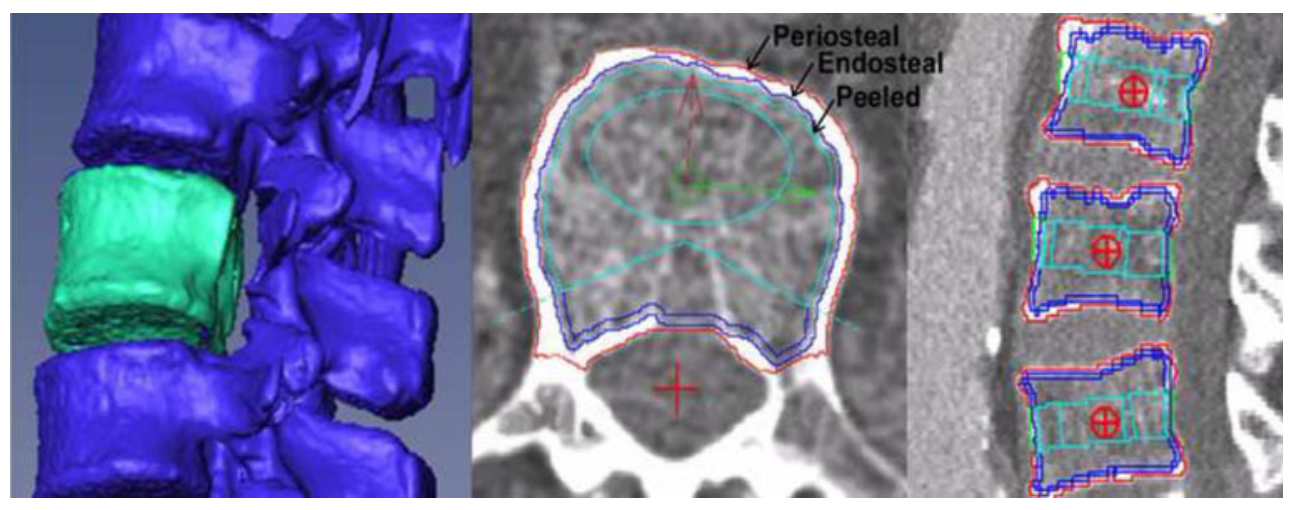

Fig. 1 Volumetric quantitative CT of the lumbar spine with automated anatomic coordinates outlining the periosteal, endosteal, and juxtaendosteal ("peeled") contours of the vertebral body. Several different volume of interests (VOIs) can be evaluated, such as the total, trabecular, peeled, elliptical, and Pacman VOIs in the axial plane, as well as the superior, mid-vertebral, and inferior VOIs in the sagittal plane. (Image courtesy of Klaus Engelke)

the antiresorptive agent (alendronate) with a bone-forming agent (teriparatide) over an 18-month period, both treatments improved vertebral body strength, but the effect was more pronounced with teriparatide, which preferentially improved volumetric density and strength of the trabecular component [34]. This enhanced effect of teriparatide was evident only on finite element modeling and not on DXA or QCT total integral density data [34]. Finite element modeling revealed how treatment with teriparatide over 2 years improved vertebral body strength by as much as

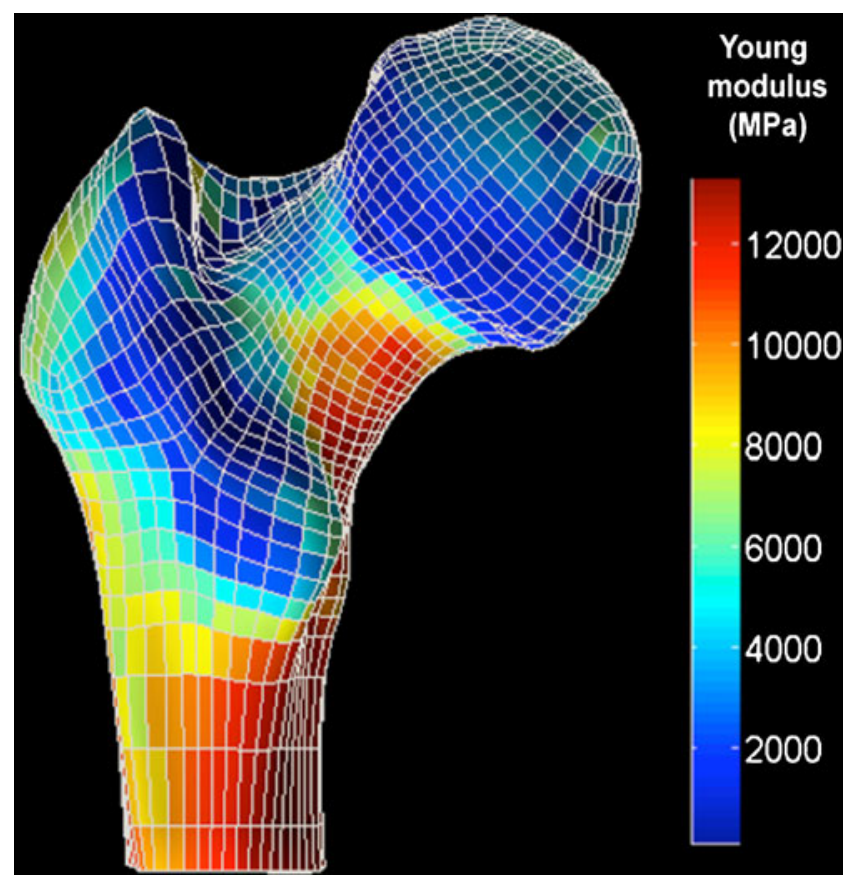

Fig. 2 Volumetric quantitative CT provides a basis for finite element analysis of the proximal femur. Note how stress distribution as related to color code is highest along the inferomedial aspect of the femoral neck and proximal shaft. (Image courtesy of Klaus Engelke) 
$30 \%$ during simulated compression and bending [35•]. This was a much better improvement in bone strength than predicted by density change alone [35•].

Similarly, for the proximal femur, FEA analysis of vQCT data can be used clinically to study longitudinal changes in bone strength and relate these to concurrent structural changes. Comparing aged-related changes in femoral strength with femoral neck BMDa, decreases in femoral strength with aging $(55 \%$ decrease in women, $39 \%$ decrease in men) were greater than for femoral neck BMDa (26\% decrease in women, $21 \%$ decrease in men). Femoral strength declined notably for women during their mid-40s and for men during their mid-50s [36••]. Also, the prevalence of "low femoral strength" $(<3,000 \mathrm{~N})$ was much higher than that of BMD-based "osteoporosis" [36••]. This is notable because femoral strength assessment by FEA has been shown to be more predictive of proximal femoral fracture than BMDa [37••]. FEA also has been applied to assess treatment effects in the proximal femur. Treatment of severely osteoporotic postmenopausal women with teriparatide for 24 months resulted in a significantly improved femoral buckling ratio, not at 12 months, but at 24 months, while bending strength parameters (ie, section modulus) also improved compared with baseline [38•]. These bone strength indices were associated with an increase $(4.3 \%)$ in proximal femoral cortical cross-sectional area due to endosteal rather than periosteal bone apposition [38•]. Future studies will help confirm whether this improved treatment evaluation afforded by FEA also translates into improved fracture prediction.

\section{Peripheral Quantitative CT}

Dedicated peripheral QCT (pQCT) systems with a resolution comparable to that of MDCT $(500 \mu \mathrm{m})$ have been available - though not widely used-since 1990 to examine the peripheral skeleton. These systems confer a smaller effective radiation dose and are particularly useful for studying cortical bone changes in metabolic bone disorders because the distal radius contains more cortical bone than the vertebral body. A pQCT study has shown how cortical bone loss begins during middle age for women, though much later in men (after age 75 years), whereas trabecular bone loss begins in early adulthood for both sexes [39]. By age 50 years, approximately $40 \%$ of total lifetime trabecular loss has occurred, although only $15 \%$ of cortical bone loss has occurred by that point [39]. As pQCT units use low-power $\mathrm{x}$-ray tubes, these examinations are slow, with a tendency toward motion artefact. With this limitation in mind, the feasibility of using clinical CT scanners with a dedicated forearm phantom as an alternative to $\mathrm{pQCT}$ has been investigated [40•]. BMD data (from both cortical and trabecular bone) as well as geometric data (cortical thickness, cortical BMD, and density-weighted polar moment of inertia [a measure of bone strength]) are obtainable from the diaphysis to ultradistal radius with good accuracy and very good precision in less than $30 \mathrm{~s}$, compared with about $4 \mathrm{~min}$ for dedicated pQCT systems [40॰]. Applying a clinical CT scanner to study the effect of denosumab, a human monoclonal antibody that inhibits RANKL (receptor activator of nuclear factor- $\mathrm{KB}$ ligand), on the distal radius of osteopenic postmenopausal women revealed a positive effect on both the cortical and trabecular bone compartments, as well as bone strength - estimated by densityweighted polar moment of inertia-compared with placebo [41]. This indicates how a clinical CT system can be used as a good alternative to pQCT systems to
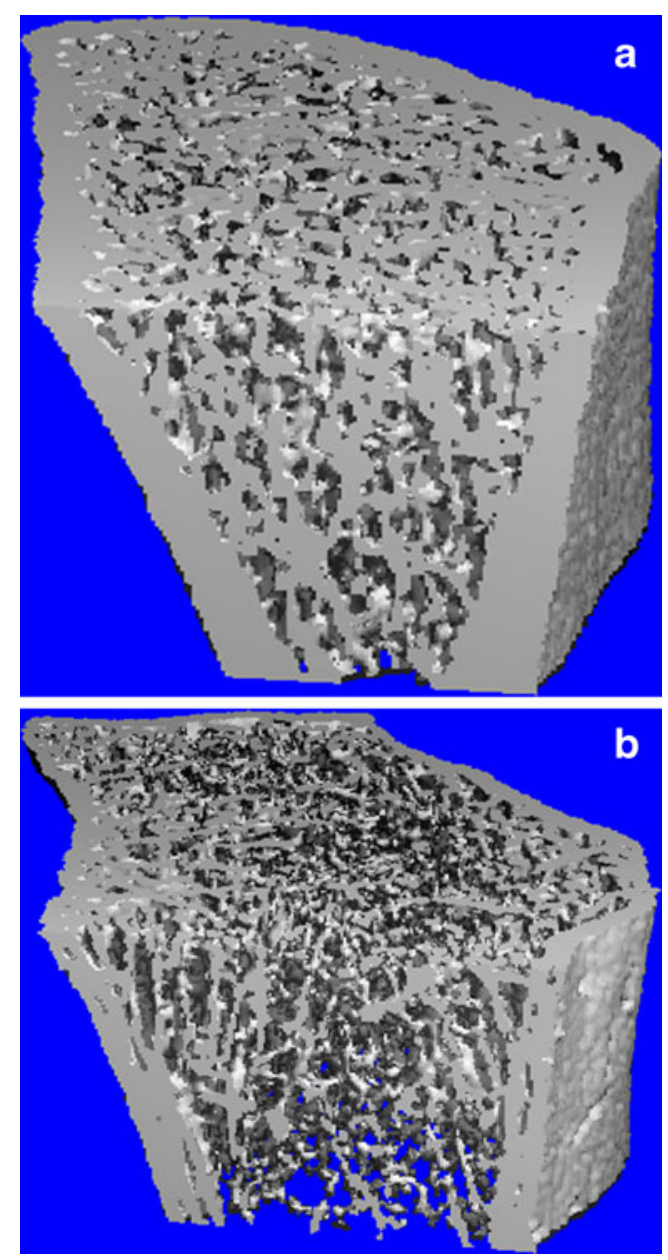

Fig. 3 High-resolution peripheral quantitative CT (XtremeCT; Scanco Medical, Brüttisellen, Switzerland) of the distal radius. a, Systemic lupus erythematosus (SLE) patient on steroids without vertebral fracture. b, SLE patient on steroids with vertebral fracture. Note the relative deterioration in trabecular architecture and thinning with increased porosity of cortex in the patient with vertebral fracture compared with the patient without vertebral fracture. (Image courtesy of Edmund Li) 
assess the distal forearm. This also applies to the experimental study of small animals [42•].

\section{High-Resolution Peripheral Quantitative CT}

Bridging the clinical need for an imaging modality with lower radiation dose, yet with better spatial resolution is the high-resolution pQCT scanner (HR-pQCT) (XtremeCT, Scanco Medical, Brüttisellen, Switzerland). This can evaluate bone architecture in the distal radius and tibia with an isotropic voxel of $82 \mu^{3}$ (spatial resolution $120 \mu \mathrm{m}$ ), though with an effective radiation dose about 0.1 of that for MDCT of the more central skeleton. Even at this much improved resolution, individual trabeculae $(50-150 \mu \mathrm{m}$ thick) still fill only one to two voxels, leading to considerable partial volume averaging. The result is that trabecular spacing $(400-800 \mu \mathrm{m}$ wide) can be accurately assessed, but modest limitations still exist in measuring trabecular number and thickness (50$150 \mu \mathrm{m}$ ), although these difficulties are considerably less than with MDCT. In 2005, the first published clinical study assessing HR-pQCT found that postmenopausal women had lower BMD, trabecular number, and cortical thickness compared with premenopausal women at the distal radius and tibia, although spine and hip BMDa were similar [43]. Compared with healthy controls, HR-pQCT revealed increased cortical porosity in postmenopausal osteopenic/ osteoporotic women [44] and also in diabetics, especially those with osteoporotic fracture [45]. Comparing systemic lupus erythematosus patients on steroids with and without vertebral fracture, no BMDa differences were apparent on spine DXA examination, although several key cortical and trabecular structural parameter differences were apparent on HR-pQCT examination of the distal radius (Fig. 3) [46•]. HR-pQCT is clearly a useful modality for assessing changes in cortical and trabecular bone structural parameters, with a precision of about $2 \%$ to $5 \%$ [43]. The main limitations of HR-pQCT are that it requires a dedicated scanner, is confined to examination of the distal forearm and leg, has some difficultly with registration in the $\mathrm{Z}$ plane (longitudinal plane), and should take into consideration the expected difference among individuals of short or long radial or tibial bone length.

\section{MRI}

Although the spatial resolution of MRI is less than that of other techniques, such as MDCT, it does possess several other advantages that will ensure its increasing deployment to bone research for the foreseeable future. In addition to not using ionizing radiation, it can directly acquire images in any plane de novo and has the potential to derive functional information from bone and bone marrow not readily available with other imaging techniques. Since its first clinical bone applications in 1977 [1•, 47], improvements in coil technology, pulse sequencing, analytical techniques, and gantry hardware have advanced the usefulness of MRI in all aspects of bone imaging, including osteoporosis research (Fig. 4). For example, medullary canal signal-to-noise ratio is up to 16 times better with 3.0-T magnetic resonance (MR) than with 1.5-T MR [48], which contributes to in vitro trabecular bone architecture being better delineated at $3.0 \mathrm{~T}$ than $1.5 \mathrm{~T}$ using micro-CT as a reference standard [49].

Most MR-based analyses of bone architecture have been performed on the distal radius, the distal tibia, and the calcaneus because these sites contain more trabecular bone and are more easily evaluated with MR surface coils. As the in vivo, in-plane resolution achievable with MRI is 200 to $300 \mu \mathrm{m}$, it is not possible to delineate individual trabeculae. Nevertheless, apparent trabecular structural parameters derived from MRI correlate quite favorably with those derived from higher-resolution techniques such as histology or micro-CT [50, 51].

Fig. 4 High-resolution in vivo MRI of the distal radius and ulna. The circle in the midportion of the distal radius represents an area selected for virtual core biopsy as shown. (Image courtesy of Felix Wehrli)
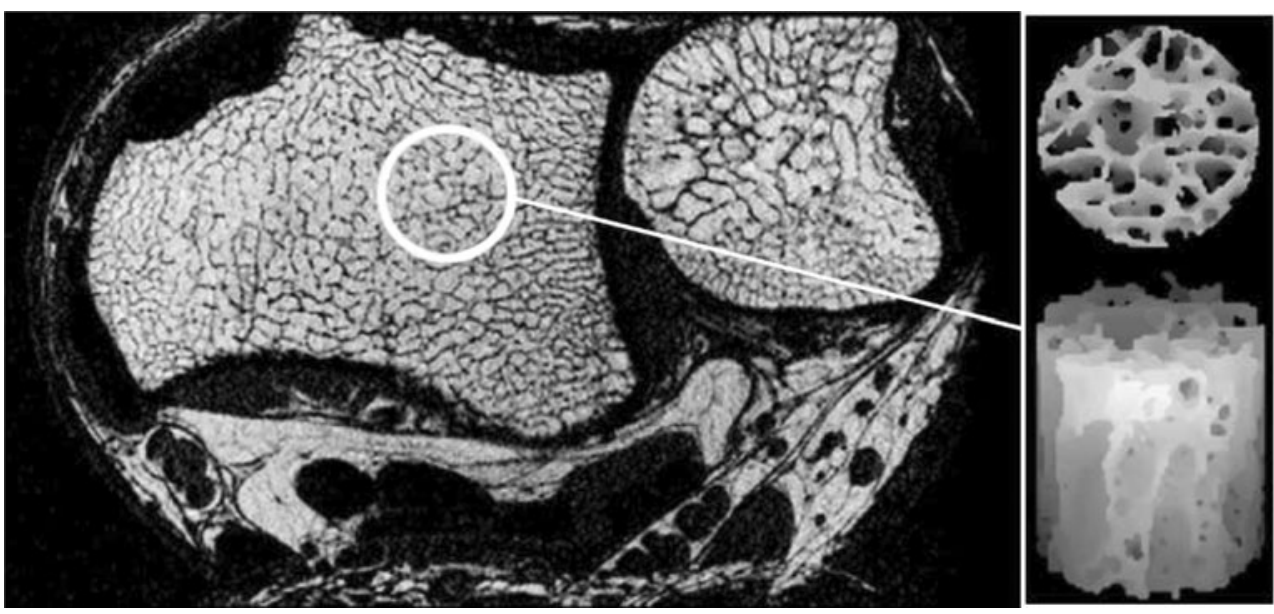
However, absolute structural parameters from HR-pQCT and high-resolution MRI were not found to be comparable $(P<0.0001)$, with trabecular bone fraction and trabecular thickness being three to four times larger with MRI than with HR-pQCT [52].

MRI has the potential to examine physiologic parameters within bone and bone marrow beyond the mineralized component [1•]. Applying sequences with ultra-short echo times allows detection of molecules with ultra-short T2 relaxation times, such as water, within highly ordered tissues such as cortical bone or tendon. Quantification of the emitted signal provides an index of cortical bone water content as a potential parameter of bone strength. Using a technique validated in sheep and human tibial specimens, water content of the tibial diaphysis was higher in postmenopausal women than premenopausal women and much higher in women with chronic renal failure on hemodialysis, even though no difference in tibial volumetric BMD was found among the three groups [53].

At the moment, there is no noninvasive imaging modality capable of imaging bone matrix (ie, the collagenous lattice onto which the mineralized component [mainly calcium phosphate] of bone is deposited). This collagen component and the degree of collagen cross-linking are important for bone strength. Using a specifically designed reference phantom (simulating bone matrix composition of collagen) and a specifically designed MR pulse sequence known as WASPI (waterand fat-suppressed proton projection MRI), porcine cortical and trabecular bone samples were evaluated [54]. A very high correlation $\left(r^{2}=0.98\right.$ and 0.95$)$ between WASPI intensity values and gravimetric and amino acid analyses, respectively, was observed, indicating that WASPI sequences appear capable of measuring the bone matrix mass density in $\mathrm{g} / \mathrm{cm}^{3}$ [54].

By quantifying the phosphorus component of bone mineral, phosphorus spectroscopy $\left({ }^{31} \mathrm{PMRS}\right)$ can be used to measure $\mathrm{BMD}$. The ratio of $\mathrm{BMD} /$ bone matrix density therefore provides a measure of the degree of "bone matrix mineralization" [54]. Applying these techniques, clear differences in the bone matrix density and degree of bone matrix mineralization were found comparing ovarectomized rats, partially nephrectomized rats, and control rats [55••]. For the bone cortex, no significant differences were observed between controls and ovarectomized rats. However, for nephrectomized rats, MR study revealed a clear reduction in cortical BMD (decreased by $22 \%$ ), bone matrix density (decreased by $10 \%$ ), and extent of bone matrix mineralization (decreased by $12 \%$ ). For trabecular bone, BMD was seen to be reduced in ovarectomized rats (decreased by $40 \%$ ) and nephrectomized rats (decreased by $27 \%$ ), although only the ovarectomized rats showed a reduction in bone matrix density (decreased by $38 \%$ ), while instead the nephrectomized rats showed a reduction in extent of bone matrix mineralization (decreased by $26 \%$ ) compared with controls [55••]. It is also technically feasible to undertake WASPI imaging in humans, offering exciting prospects for clinical research [56].

\section{Vertebral Fracture Recognition}

The quest for ever-finer determination of bone structure and quality should not override the day-to-day recognition of another much more tangible and immediately clinically relevant parameter of bone quality. The presence of an insufficiency fracture, of which vertebral fracture is by far the most frequent, provides indisputable evidence of reduced bone strength irrespective of BMD or other measurement. However, in clinical practice, vertebral and other insufficiency fractures frequently go unnoticed or unreported [57, 58]. The incentive of the "vertebral fracture initiative" is to promote recognition among clinical radiologists and clinicians of the importance of vertebral fracture and to ensure that such fractures, whether they be on chest radiography, abdominal radiography, or thoracoabdominal CT studies, are reported in a standardized, unambiguous fashion [59-61].

\section{Conclusions}

The past 3 to 4 years have seen the exponential growth of bone imaging across several complementary modalities continue from its infancy more than 30 years ago. These recent advances in bone imaging - both experimental and practical-have provided significant inroads into the understanding of the prevalence, disease progression, and pathophysiology of metabolic bone disease, particularly osteoporosis. This newfound knowledge has already begun to translate into everyday clinical practice. This trend will continue such that in the not-too-distant future, modifications of new imaging modalities will become more widely available and thus will allow for greatly improved recognition, treatment, and monitoring of the osteoporotic process.

Disclosure Dr. Genant has served on a board for and owned stock/ stock options in SYNARC and has served as a consultant for, received honoraria from, received payment for development of educational presentations (including service on speakers' bureaus) from, and had travel/accommodations expenses covered/reimbursed by Merck \& Co., Novartis, Servier, Bristol-Myers Squibb, GlaxoSmithKline, Roche, Eli Lilly and Company, Genentech, Amgen, and Ono Pharmaceutical Co. Dr. Griffith reported no potential conflict of interest relevant to this article. 
Open Access This article is distributed under the terms of the Creative Commons Attribution Noncommercial License which permits any noncommercial use, distribution, and reproduction in any medium, provided the original author(s) and source are credited.

\section{References}

Papers of particular interest, published recently, have been highlighted as:

- Of importance

•- Of major importance

1. - Link TM. The Founder's Lecture 2009: advances in imaging of osteoporosis and osteoarthritis. Skeletal Radiol. 2010;39:943-55. This is a comprehensive, easy-to understand overview of recent advances in bone imaging of osteoporosis with a historical perspective.

2. Hernandez CJ, Keaveny TM. A biomechanical perspective on bone quality. Bone. 2006;39:1173-81.

3. Cummings SR, Cawthon PM, Ensrud KE, Cauley JA, Fink HA, Orwoll ES, et al. BMD and risk of hip and nonvertebral fractures in older men: a prospective study and comparison with older women. J Bone Miner Res. 2006;21:1550-6.

4. Rivadeneira F, Zillikens MC, De Laet CE, Hofman A, Uitterlinden AG, Beck TJ, et al. Femoral neck BMD is a strong predictor of hip fracture susceptibility in elderly men and women because it detects cortical bone instability: the Rotterdam Study. J Bone Miner Res. 2007;22:1781-90.

5. Johnell O, Kanis JA, Oden A, Johansson H, De Laet C, Delmas P, et al. Predictive value of BMD for hip and other fractures. J Bone Miner Res. 2005;20:1185-94. Erratum in: J Bone Miner Res. 2007;22:774.

6. Lewiecki EM, Gordon CM, Baim S, Leonard MB, Bishop NJ, Bianchi ML, et al. International society for clinical densitometry 2007 adult and pediatric official positions. Bone. 2008;43:111521

7. Kanis JA, Johansson H, Oden A, McCloskey EV. Assessment of fracture risk. Eur J Radiol. 2009;71:392-7.

8. Dasher LG, Newton CD, Lenchik L. Dual X-ray absorptiometry in today's clinical practice. Radiol Clin North Am. 2010;48:541-60.

9. Genant HK, Li J, Wu CY, Shepherd JA. Vertebral fractures in osteoporosis: a new method for clinical assessment. J Clin Densitom. 2000;3:281-90.

10. McCloskey EV, Vasireddy S, Threlkeld J, Eastaugh J, Parry A, Bonnet $\mathrm{N}$, et al. Vertebral fracture assessment (VFA) with a densitometer predicts future fractures in elderly women unselected for osteoporosis. J Bone Miner Res. 2008;23:1561-8.

11. Rüegsegger P, Elsasser U, Anliker M, Gnehm H, Kind H, Prader A. Quantification of bone mineralization using computed tomography. Radiology. 1976;121:93-7.

12. Genant HK, Boyd DP. Quantitative bone mineral analysis using dual energy computed tomography. Invest Radiol. 1977;12:545-51.

13. Genant HK, Cann CE, Pozzi-Mucelli RS, Kanter AS. Vertebral mineral determination by quantitative computed tomography: clinical feasibility and normative data. J Comput Assist Tomogr. 1983;7:554

14. Heuck AF, Block J, Glueer CC, Steiger P, Genant HK. Mild versus definite osteoporosis: comparison of bone densitometry techniques using different statistical models. J Bone Miner Res. 1989;4:891-900.

15. Black DM, Greenspan SL, Ensrud KE, Palermo L, McGowan JA, Lang TF, et al. PaTH Study Investigators. The effects of parathyroid hormone and alendronate alone or in combination in postmenopausal osteoporosis. N Engl J Med. 2003;349:1207-15.

16. McCollough $\mathrm{CH}$, Guimarães L, Fletcher JG. In defense of body CT. AJR Am J Roentgenol. 2009;193:28-39.

17. - Mueller DK, Kutscherenko A, Bartel H, Vlassenbroek A, Ourednicek P, Erckenbrecht J. Phantom-less QCT BMD system as screening tool for osteoporosis without additional radiation. Eur J Radiol. 2010 Mar 9. [Epub ahead of print]. This study presents a comprehensive analysis of the precision and clinical applicability of phantom-less QCT analyses that can be applied in crosssectional observational studies even retrospectively, but are not recommended for longitudinal study.

18. Budoff MJ, Hamirani YS, Gao YL, Ismaeel H, Flores FR, Child J, et al. Measurement of thoracic bone mineral density with quantitative CT. Radiology. 2010;257:434-40.

19. Bauer JS, Henning TD, Müeller D, Lu Y, Majumdar S, Link TM. Volumetric quantitative CT of the spine and hip derived from contrast-enhanced MDCT: conversion factors. AJR Am J Roentgenol. 2007;188:1294-301.

20. Lee CI, Forman HP. What we can and cannot see coming. Radiology. 2010;257:313-14.

21. Rachidi M, Marchadier A, Gadois C, Lespessailles E, Chappard C, Benhamou CL. Laws' masks descriptors applied to bone texture analysis: an innovative and discriminant tool in osteoporosis. Skeletal Radiol. 2008;37:541-8.

22. Issever AS, Link TM, Kentenich M, Rogalla P, Burghardt AJ, Kazakia GJ, et al. Assessment of trabecular bone structure using MDCT: comparison of 64- and 320-slice CT using HR-pQCT as the reference standard. Eur Radiol. 2010;20:458-68.

23. Graeff C, Timm W, Nickelsen TN, Farrerons J, Marin F, Barker C, et al. Monitoring teriparatide-associated changes in vertebral microstructure by high-resolution $\mathrm{CT}$ in vivo: results from the EUROFORS study. J Bone Miner Res. 2007;22:1426-33.

24. Kang Y, Engelke K, Fuchs C, Kalender WA. An anatomic coordinate system of the femoral neck for highly reproducible BMD measurements using 3D QCT. Comput Med Imaging Graph. 2005;29:533-41.

25. Engelke K, Mastmeyer A, Bousson V, Fuerst T, Laredo JD, Kalender WA. Reanalysis precision of 3D quantitative computed tomography (QCT) of the spine. Bone. 2009;44:566-72.

26. Bousson V, Le Bras A, Roqueplan F, Kang Y, Mitton D, Kolta S, et al. Volumetric quantitative computed tomography of the proximal femur: relationships linking geometric and densitometric variables to bone strength. Role for compact bone. Osteoporos Int. 2006; 17:855-64.

27. •• Engelke K, Fuerst T, Dasic G, Davies RY, Genant HK. Regional distribution of spine and hip QCT BMD responses after one year of once-monthly ibandronate in postmenopausal osteoporosis. Bone. 2010;46:1626-32. This study illustrates the benefit of applying automated anatomic coordinate systems to facilitate CT image analysis. Comparison of predetermined anatomic areas allows appreciation of how different bone areas may be responding to treatment and how improvements to those more structurally relevant areas helps explain observed changes in bone strength following treatment.

28. Crawford RP, Cann CE, Keaveny TM. Finite element models predict in vitrovertebral body compressive strength better than quantitative computed tomography. Bone. 2003;33:744-50.

29. Keyak JH, Rossi SA, Jones KA, Skinner HB. Prediction of femoral fracture load using automated finite element modeling. J Biomech. 1998;31:125-33.

30. MacNeil JA, Boyd SK. Bone strength at the distal radius can be estimated from high-resolution peripheral quantitative computed tomography and the finite element method. Bone. 2008;42:1203-13.

31. •- Christiansen BA, Kopperdahl DL, Kiel DP, Keaveny TM, Bouxsein ML. Mechanical contributions of the cortical and 
trabecular compartments contribute to differences in age-related changes in vertebral body strength in men and women assessed by QCT-based finite element analysis. J Bone Miner Res. 2010 Nov 18. [Epub ahead of print]. This study explored how structural changes influence bone strength as assessed by FEA. It showed how increased vertebral body fragility in older women occurs as a result of trabecular thinning being accompanied by cortical thinning, while in men, vertebral cortical bone is less affected. In other words, it showed how males and females lose bone differently with age, with females having a relatively greater degree of vertebral body cortical bone loss that contributes to an increased prevalence of vertebral fracture.

32. - Melton LJ 3rd, Riggs BL, Keaveny TM, Achenbach SJ, Kopperdahl D, Camp JJ, et al. Relation of vertebral deformities to bone density, structure, and strength. J Bone Miner Res. 2010;25:1922-30. This study showed how structural and strength parameters are all reduced in individuals with more severe vertebral body fractures rather than those with milder vertebral fractures. Vertebral fracture severity is an additional indicator of bone strength, and mild vertebral deformities do represent early vertebral fractures.

33. Mawatari T, Miura H, Hamai S, Shuto T, Nakashima Y, Okazaki $\mathrm{K}$, et al. Vertebral strength changes in rheumatoid arthritis patients treated with alendronate, as assessed by finite element analysis of clinical computed tomography scans: a prospective randomized clinical trial. Arthritis Rheum. 2008;58:3340-9.

34. Keaveny TM, Donley D, Hoffmann P, Mitlak B, Glass E, San Martin J. Effects of teriparatide and alendronate on vertebral strength as assessed by finite element modeling of QCT scans in women with osteoporosis. J Bone Miner Res. 2007;22:14957.

35. - Graeff C, Chevalier Y, Charlebois M, Varga P, Pahr D, Nickelsen $\mathrm{TN}$, et al. Improvements in vertebral body strength under teriparatide treatment assessed in vivo by finite element analysis: results from the EUROFORS study. J Bone Miner Res. 2009;24:1672-80. This study showed how finite element modeling of volumetric CT data can be used to show improvements in vertebral bone strength over and above those predicated by density change alone. However, it does conclude that further study is required to confirm that this improved estimation of vertebral body strength translates into improved prediction of vertebral fracture.

36. •• Keaveny TM, Kopperdahl DL, Melton LJ 3rd, Hoffmann PF, Amin S, Riggs BL, et al. Age-dependence of femoral strength in white women and men. J Bone Miner Res. 2010;25:994-1001. This study used FEA as a much more direct in vivo measure of bone strength to plot changes in proximal femoral strength with aging. Proximal femoral strength declines with age, particularly in women-much more so than would be predicted on the basis of BMDa changes. It also showed how low femoral strength is much more prevalent in older adults than osteoporosis as defined using $B M D a$.

37. • Orwoll ES, Marshall LM, Nielson CM, Cummings SR, Lapidus J, Cauley JA, et al.; Osteoporotic Fractures in Men Study Group. Finite element analysis of the proximal femur and hip fracture risk in older men. J Bone Miner Res. 2009;24:475-83. This is the first prospective study to evaluate the use of FEA in predicting proximal femoral fracture. Femoral strength assessment by FEA was more predictive of proximal femoral fracture than BMDa.

38. - Borggrefe J, Graeff C, Nickelsen TN, Marin F, Glüer CC. Quantitative computed tomographic assessment of the effects of 24 months of teriparatide treatment on 3D femoral neck bone distribution, geometry, and bone strength: results from the EUROFORS study. J Bone Miner Res. 2010;25:472-81. This study used FEA to study the effect of treatment on proximal femoral bone strength. Bone strength indices improved with treatment associated with an increase in proximal femoral cortical thickness.

39. Riggs BL, Melton LJ, Robb RA, Camp JJ, Atkinson EJ, McDaniel L, et al. A population-based assessment of rates of bone loss at multiple skeletal sites: evidence for substantial trabecular bone loss in young adult women and men. J Bone Miner Res. 2008;23:205-14.

40. • Engelke K, Libanati C, Liu Y, Wang H, Austin M, Fuerst T, et al. Quantitative computed tomography (QCT) of the forearm using general purpose spiral whole-body CT scanners: accuracy, precision and comparison with dual-energy X-ray absorptiometry (DXA). Bone. 2009;45:110-8. This study showed how clinical CT units with a dedicated forearm phantom can be used as a good alternative to $p Q C T$.

41. Genant HK, Engelke K, Hanley DA, Brown JP, Omizo M, Bone $\mathrm{HG}$, et al. Denosumab improves density and strength parameters as measured by QCT of the radius in postmenopausal women with low bone mineral density. Bone. 2010;47:131-9.

42. - Wang YX, Griffith JF, Zhou H, Choi KC, Hung VW, Yeung DK, et al. Rat lumbar vertebrae bone densitometry using multidetector CT. Eur Radiol. 2009;19:882-90. This study showed how clinical $C T$ units can be used as a good alternative to $p Q C T$ when studying longitudinal density changes in small animals.

43. Boutroy S, Bouxsein ML, Munoz F, Delmas PD. In vivo assessment of trabecular bone microarchitecture by highresolution peripheral quantitative computed tomography. J Clin Endocrinol Metab. 2005;90:6508-15.

44. Nishiyama KK, Macdonald HM, Buie HR, Hanley DA, Boyd SK. Postmenopausal women with osteopenia have higher cortical porosity and thinner cortices at the distal radius and tibia than women with normal aBMD: an in vivo HR-pQCT study. J Bone Miner Res. 2010;25:882-90.

45. Burghardt AJ, Dais KA, Masharani U, Link TM, Majumdar S. In vivo quantification of intra-cortical porosity in human cortical bone using hr-pQCT in patients with type II diabetes. J Bone Miner Res. 2008;23:S450.

46. - Li EK, Zhu TY, Tam LS, Hung VW, Griffith JF, Li TK, et al. Bone microarchitecture assessment by high-resolution peripheral quantitative computed tomography in patients with systemic lupus erythematosus taking corticosteroids. J Rheumatol. 2010;37:1473-9. This study showed how structural parameters evident on HR-pQCT of the distal radius proved more discriminatory at distinguishing between systemic lupus erythematosus patients on steroids with and without vertebral fracture than spinal BMDa.

47. Hinshaw WS, Bottomley PA, Holland GN. Radiographic thinsection image of the human wrist by nuclear magnetic resonance. Nature. 1977;270(22-29):722-3.

48. Ludescher B, Martirosian P, Lenk S, Machann J, Dammann F, Schick F, et al. High-resolution magnetic resonance imaging of trabecular bone in the wrist at 3 tesla: initial results. Acta Radiol. 2005;46:306-9.

49. Phan CM, Matsuura M, Bauer JS, Dunn TC, Newitt D, Lochmueller EM, et al. Trabecular bone structure of the calcaneus: comparison of MR imaging at 3.0 and $1.5 \mathrm{~T}$ with micro-CT as the standard of reference. Radiology. 2006;239:488-96.

50. Wehrli FW, Gomberg B, Saha P, Song H, Hwang S, Snyder P. Digital topological analysis of in vivo magnetic resonance microimages of trabecular bone reveals structural implications of osteoporosis. J Bone Miner Res. 2001;16:1520-31.

51. Wehrli FW, Leonard MB, Saha PK, Gomberg BR. Quantitative high-resolution magnetic resonance imaging reveals structural implications of renal osteodystrophy on trabecular and cortical bone. J Magn Reson Imaging. 2004;20:83-9.

52. Kazakia GJ, Hyun B, Burghardt AJ, Krug R, Newitt DC, de Papp AE, et al. In vivo determination of bone structure in 
postmenopausal women: a comparison of HR-pQCT and highfield MR imaging. J Bone Miner Res. 2008;23:463-74.

53. Techawiboonwong A, Song HK, Wehrli FW. In vivo MRI of submillisecond $\mathrm{T}(2)$ species with two-dimensional and threedimensional radial sequences and applications to the measurement of cortical bone water. NMR Biomed. 2008;21:59-70.

54. Cao H, Ackerman JL, Hrovat MI, Graham L, Glimcher MJ, Wu Y. Quantitative bone matrix density measurement by water- and fatsuppressed proton projection MRI (WASPI) with polymer calibration phantoms. Magn Reson Med. 2008;60:1433-43.

55. •- Cao H, Nazarian A, Ackerman JL, Snyder BD, Rosenberg AE, Nazarian RM, et al. Quantitative (31)P NMR spectroscopy and (1) $\mathrm{H}$ MRI measurements of bone mineral and matrix density differentiate metabolic bone diseases in rat models. Bone. 2010;46:1582-90. This study showed for the first time how an MR-based technique can be used to noninvasively quantify the amount of bone matrix (ie, the collagenous component of bone) and accordingly distinguish between experimentally induced osteoporosis and osteomalacia.
56. Wu Y, Hrovat MI, Ackerman JL, Reese TG, Cao H, Ecklund K, et al. Bone matrix imaged in vivo by water- and fat-suppressed proton projection MRI (WASPI) of animal and human subjects. J Magn Reson Imaging. 2010;31:954-63.

57. Gehlbach S, Bigelow C, Heimisdottir M, May S, Walker M, Kirkwood J. Recognition of vertebral fracture in a clinical setting. Osteoporos Int. 2000;11:577-82.

58. Williams AL, Al-Busaidi A, Sparrow PJ, Adams JE, Whitehouse RW. Under-reporting of osteoporotic vertebral fractures on computed tomography. Eur J Radiol. 2009;69:179-83.

59. Genant HK, Wu CY, van Kuijk C, Nevitt MC. Vertebral fracture assessment using a semiquantitative technique. J Bone Miner Res. $1993 ; 8: 1137-48$.

60. Grigoryan M, Guermazi A, Roemer FW, Delmas PD, Genant HK. Recognizing and reporting osteoporotic vertebral fractures. Eur Spine J. 2003;12 Suppl 2:S104-12.

61. Link TM, Guglielmi G, van Kuijk C, Adams JE. Radiologic assessment of osteoporotic vertebral fractures: diagnostic and prognostic implications. Eur Radiol. 2005;15:1521-32. 